\title{
Simulation modelling of Carrapateena Sub Level Cave production level performance
}

\author{
D Hronsky OZ Minerals, Australia
}

S Mariager OZ Minerals, Australia

S Donaldson Polymathian, Australia

L Bobbermen Polymathian, Australia

\author{
C Eustace Polymathian, Australia
}

\begin{abstract}
Carrapateena is a copper-gold deposit hosted in a brecciated granite complex, located approximately 460 $\mathrm{km}$ north of Adelaide, South Australia. The deposit will be mined using the sub level cave (SLC) mining method at a rate of $4.25 \mathrm{Mtpa}$ for an estimated 20 years. When entering its second phase of construction and planning prior to production commencement, the mining teams at Carrapateena were beginning to shift focus from high-level long-term business planning, into more detailed execution planning, which involved the establishment of certain operational philosophies for the production levels. These philosophies needed to consider the many varied elements that influence the production plan. Each of these elements, including both mining equipment and tasks have diverse operational rules, and with a large and complex operation to plan for, it was important to clearly understand the interactions between the elements and the effect on the production system as a whole. To understand these complexities, OZ Minerals approached Polymathian who were able to create a detailed model of all entities and processes in the caving operation using a bespoke, discrete event simulation tool. The tool allowed planners to visualise the cave's operations over the life of a production level down to day-to-day, minute-by-minute operations. Each production process, entity and interactions between entities were modelled so planners could test various assumptions and constraints. The level of detail represented in the simulation model allowed OZ Minerals to develop an understanding of the effect of strategic and operational decisions prior to the mine going into production. The ability to validate the effect of assumptions on operational performance and test a range of scenarios enabled identification of the levers that have the biggest impact on production. This facilitated improved operations planning decisions and saved significant time and resources in planning and during operations. New staff are also able to use the visual outputs of the simulation model to understand the operational complexities on the production level and interactions that will occur between mobile equipment. The work assisted putting Carrapateena in a better position to 'hit the ground running' with reduced uncertainty around operational expectations.
\end{abstract}

\section{Introduction}

Carrapateena is a copper-gold project located approximately $160 \mathrm{~km}$ north of Port Augusta in South Australia's highly prospective Gawler Craton. The orebody is being mined using the sub-level caving methodology, consisting of 34 transverse production levels at $25 \mathrm{~m}$ sub level spacing for the top half of the orebody, and $30 \mathrm{~m}$ for the lower half. These are accessed by a main decline, with ore transported to surface via trucks initially, and then by primary crushers and conveyor belts installed on a second decline in the future. 


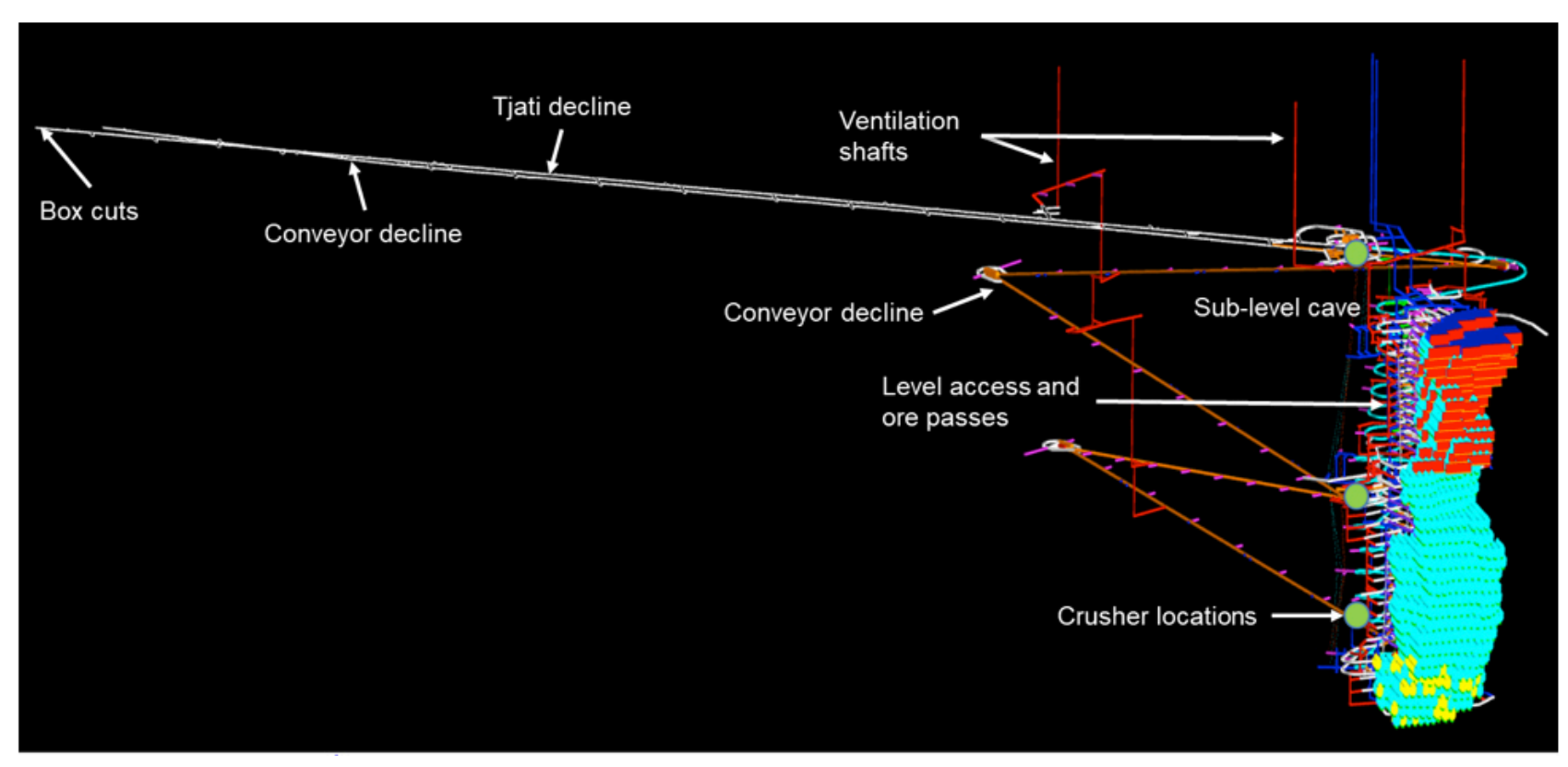

\section{Figure 1 Carrapateena mine overview}

The production level design and ring layout follows comparable modern SLC operations in Australia but differs by way of using a crows foot level layout. This layout is aimed at improving safety in design, and to support production efficiency and future automation (Mooney et al. 2020).

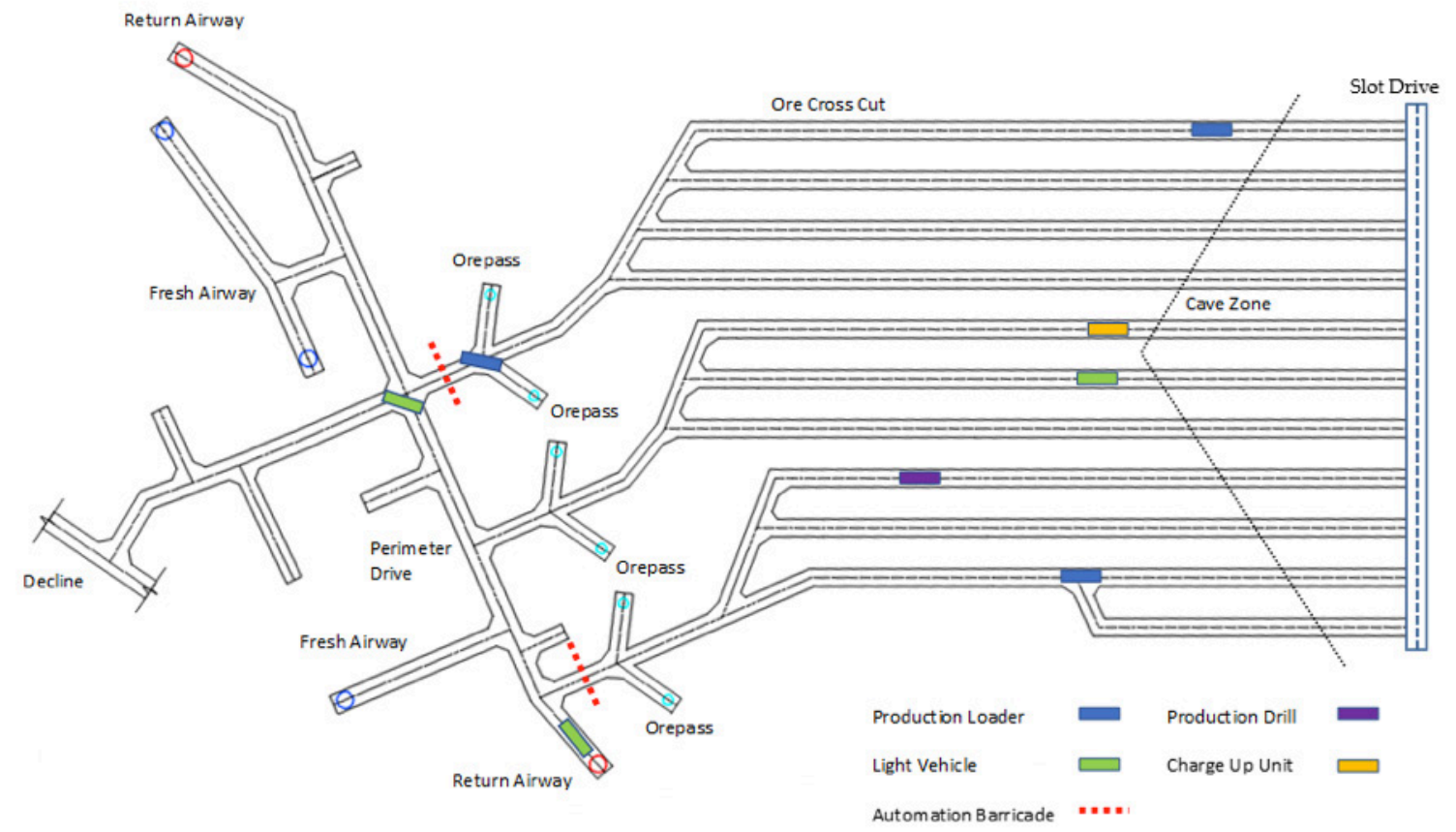

Figure 2 Carrapateena production level design

A key benefit to the crow's foot design is in the improved segregation of equipment, allowing loaders to operate more efficiently. However, the relatively small SLC footprint at Carrapateena, coupled with a fast ring turnover rate on the top three levels due to a "40/60/90 draw" (percentage of tonnes bogged vs tonnes blasted on each level), results in relatively high intensity, high interaction production level. This adds operational complexity that needs to be managed by a robust execution philosophy. 


\section{$2 \quad$ Problem statement}

Validation of productivity assumptions is an important step in mine planning stage to ensure that realistic, achievable forecasts are being produced. Traditionally these assumptions are derived from first principals and rules of thumb when no site-specific historical data is available prior to the commencement of mining. Productivity assumptions, activity durations, equipment availability and utilisation targets are input into scheduling software to determine a baseline production profile when applied to the mine design alongside task and location dependencies, resourcing, and mining strategy.

Consideration for interactions between equipment and exclusion zones between activities is often unaccounted for in these productivity assumptions, as are time-based constraints such as firing windows, crib time, daily servicing, and shift start/end times. These interactions and constraints may result in a delay / production loss that may not be appropriately accounted for if estimates are based on broad availability and utilisation assumptions. To account for this, the addition of buffers and smoothing to account for equipment interactions and delays is generally used to add a level of conservatism / realism to the plan.

Validation of productivity assumptions was critical to OZ Minerals to reduce risks associated with having an unrealistic plan. Additionally, an understanding by the technical and operational teams of all potential bottlenecks, risks and opportunities in the system was critical as it would enable solutions to be implemented into the design and operational philosophy of the mine well ahead of time.

An operational philosophy for a production level is simple enough to model at a conceptual level using scheduling software or even as a spreadsheet exercise by making productivity assumptions, however these approaches are not effective at accounting for all of the operational complexities, interactions and constraints. These detailed dynamics of the SLC production operations are simply too detailed and dynamic to represent in this manner. As such, another approach was considered.

\subsection{Mine parameters requiring assessment}

The sub-level caving process follows a chevron pattern for the permitted sequence for ring firing and bogging operations, which presents potential constraints for both activities. Progression of the cave front is led by the central crosscuts and progression of each crosscut is constrained by advancement of the neighbouring crosscut. Caving progression (ring firing) of each crosscut cannot be in advance of the precedent crosscut and cannot lag behind by more than 2 rings in order to manage stress and confinement loss (in normal operations additional lead-lag would be approved via a TARP process, but for the purposes of the simulation the most conservative number was used). Ring firing is also constrained by:

- Bogging activities: A ring cannot be charged and fired until the planned tonnes have been drawn from the preceding ring in that crosscut. For example, if bogging activities progress the draw down from a number of fired rings, but don't finish any of these rings in a particular shift, none of the crosscuts will be available for charging and firing in the next shift.

- Drilling, prep and charging activities: Rings must be drilled, prepped and charged in adjacent crosscuts in advance of firing. Drilling must be maintained 15m (3-4 rings) in advance of adjacent crosscuts and one to three rings must be charged in advance of firing in a particular crosscut (to adhere to a "pre-charging" strategy). In a normal D\&B cycle for a ring, drilling does not have precedent activities (as blocks of rings are drilled in campaigns). However, advancement of drilling in each crosscut has the potential to limit subsequent prepping, charging activities and firing in adjacent crosscuts as the drill occupies the crosscut, preventing other activities from occurring.

- Ring firing on other levels: Ring firing must lag corresponding rings on the level above by not less than 6 rings. (this constraint is not considered for simulation analysis of standalone performance of a single level). 
- Each level of the SLC is divided into two "panels"; each consisting of two crow's feet, dedicated fresh air intake and exhaust airways, and dedicated stockpiles with truck loading bays. With an aim to reduce equipment interaction, the initial production philosophy stipulated that for each shift, each panel alternates between ancillary tasks (drilling, prep, charging and firing) and bogging operations (bogging, sampling/testing).

The above constraints have potential to significantly limit both bogging activities and ring firing activities. Bogging can only proceed while there are tonnes available from firing. Decisions on which ring to bog, and which rings to fire have the potential to have a significant influence on cave progression rate on each level and overall production rate.

\section{$3 \quad$ Methodology}

Discrete event simulation (DES) modelling, the technique applied to the described problem, is used to identify and find solutions for real-world problems for complex operations. It provides an important method of operational analysis which is able to be verified, communicated, and understood. Across industries and disciplines, DES modelling provides valuable solutions by giving clear insights into complex systems.

Most business processes can be described as a sequence of discrete events. For example, a loader presents at a drawpoint, takes a bucket, trams to a stockpile, dumps load, and trams back to the drawpoint.

A DES model was developed and used to analyse the performance of production operations for the 4580 and 4555 levels of the Carrapateena SLC. The described simulation model is based on a next-event time progression approach, using seconds as the unit of time measurement.

Events typically occur at the completion of an activity such as a drilling, charging, LHD bucket filling or emptying, or arrival at a node on the network, but may also be associated with clock time such as the start or completion of a shift. The model determines the next event to occur, which denotes a change of state in the system, moves forward to the time of the next event and processes the system state. The discrete event simulation model used to analyse Carrapateena production operations included the following components:

- Entities: an object in the system which has a state and a list of events, activities or operations to perform (often described with a flow diagram). For example, production loader, drill, charge-up truck, and firing crews are entities.

- Operations: a sequence of activities that changes the state of the system on completion.

- Activity: the smallest unit of work. Activities have either a deterministic or stochastic duration. For example, a loader trams from a draw point to an intersection in 1 minute.

- Process: a sequence of events, operations, and activities in chronological order corresponding to the behaviour of an entity in the real system. For example, a loader loads, trams, dumps, trams and loads.

- Resource: a consumable object in the system, usually temporary to limit simultaneous activities. A drive has resource limit of one which is consumed while a loader is in a drive.

- State: the current position in the list of activities for an entity or system. For example, a loader is currently in the loading state and will be finished in 35 seconds.

- Event: an instantaneous occurrence that changes the state of the system. A loader finished dumping and moves to tramming state. 
Data inputs for the model were guided by the need to define resources, process and dependencies between activities. Each entity represented by the model follows a defined process. An example of an entity process flow is provided in the figure below. The figure shows the underlying processes framework that all entities in the model followed. Each entity type had their own added complexities atop this framework.

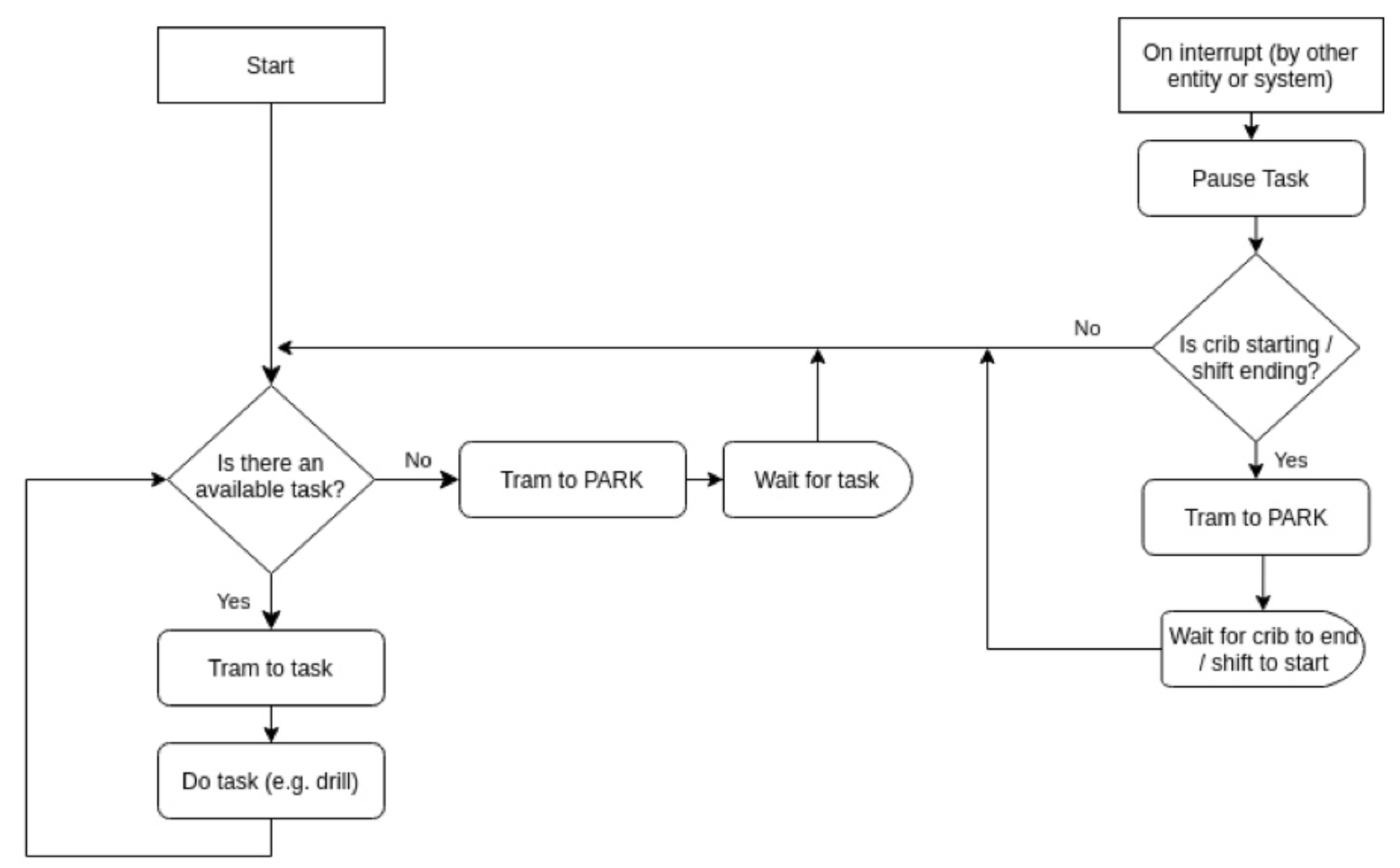

\section{Figure 3 Process flow for an entity}

To accurately model congestion, the simulation model was required to provide a representation of the "transit network". This is a connected network of tunnels and intersections through which equipment can traverse. Using this, the simulation model can determine accurate distance calculations and transit durations, and thus model congestion delays caused by interaction of multiple equipment using the network.

\subsection{Iterative base case and scenario development}

Polymathian worked with OZ Minerals to understand the data and operational rules required to represent planned operations in the simulation model. The model input data and operational assumptions were structured in a format that could be used for the model and readily modified as required. Structuring the cave rules and operational philosophy allowed conflicts and gaps to be readily identified. It also prompted detailed discussions, thinking, design modifications, and confirmation of preferred options (when faced with more than one).

After the initial data was captured an initial base case was configured, with a focus on modelling the primary entities of the production level. After the initial level layout was modelled, the following process was iteratively followed to include additional entities, and cave rules to the simulation model:

1. Run simulation scenario/s

2. Review model outputs for the simulation scenario

3. Identify the next most important model development/ refinement task to address, which was typically either or a combination of: 
a. The next entity or cave rule to be modelled

b. Enhancement to an output animation, chart or report, such that an aspect of the modelled production operation could be better understood

This process was iteratively followed until the base case scenario configuration provided a sufficiently detailed representation of future production operations and model outputs were aligned with expected cave operational performance. Several scenarios were then run with variations to the input data to test a range of operating conditions.

\section{$4 \quad$ Results}

\subsection{Numerical model outputs and visualisation of production operations}

One of the major benefits of using a detailed simulation model to analyse the system is the ability to develop detailed data to enable analysis of different aspects of the system. Since each piece of equipment is modelled, details for each event that occurs during the life of production for each level can be recorded. The simulation model is able to be configured to provide operations data in any format for every aspect of the system that affects operational performance. In addition to numerical data, animation of production operations, which shows the progression of the state of each level over time, was used to visualise operations over the life of production. The visualisation of production operations was used to identify problems either with the model representation of the mine plan, or with the mine plan itself.

Figure 4 and Figure 5 show example animation screenshots with only primary activities during stages of a ring cycle and with all the ring activities and equipment movement visible respectively. Visualisation of only the primary activities provides a clearer representation of cave progression, by only showing a handful of activity types. Visualisation of all operations as shown in Figure 5 facilitates an understanding of the complexity of production operations. In the lower panel shown in the figure, there are two drills and a charge-up operating; and in the upper panel there is a loader and a ring-sampling LV operating.

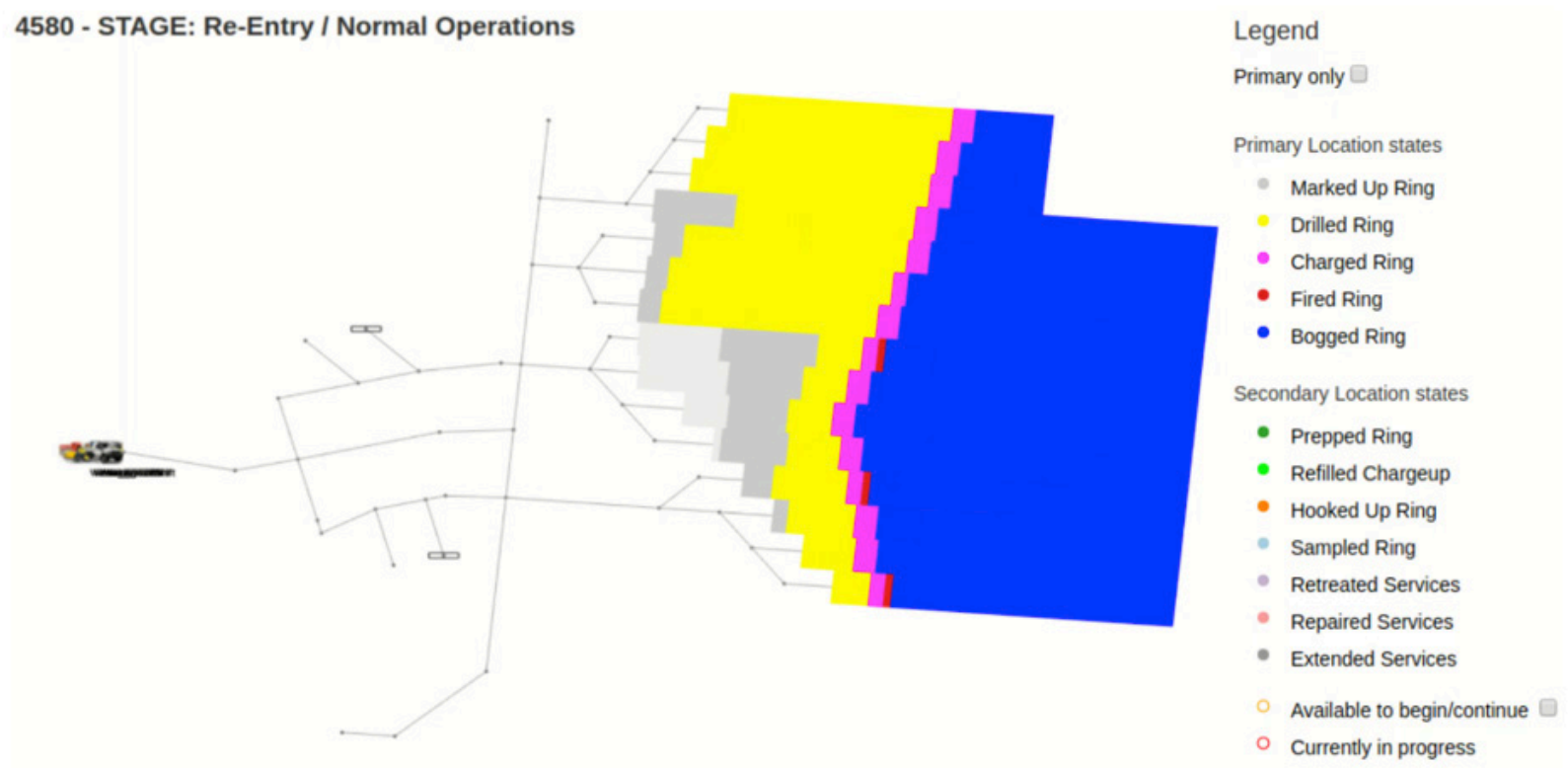

Figure 4 Example animation showing caving progression without equipment movement details 


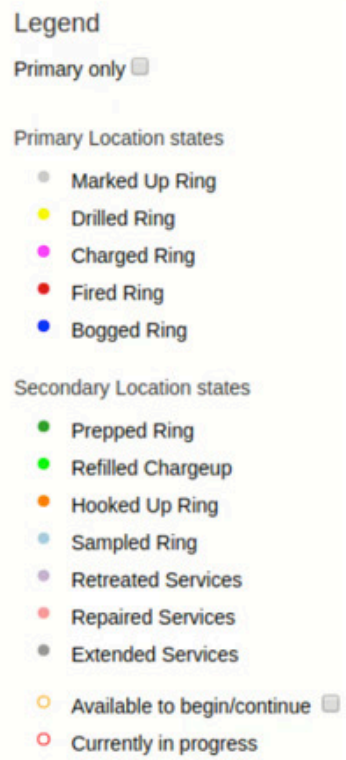

Figure 5 Example animation showing detailed movement of production and ancillary equipment

The ability to show a potential state of the mine at a given point in time, with mobile equipment operating, facilitates explanation of operational concepts. System bottlenecks are also readily apparent at each point in time by viewing the animation. Animation outputs from the model have since been shown to crews for education purposes.

\subsection{Key findings}

\subsubsection{Overall production performance}

As described in section 3, the decision logic used for task allocation to resources was simplistic, providing conservative estimates of 4580 and 4555 level production performance. These conservative production estimates aligned well with production forecasts, indicating that the forecasts were likely to be achievable for the 4580 and 4555 levels. While there are inter-level dependencies that have the potential to further constrain firing and bogging sequences were not modelled, there is an opportunity to implement improved decision logic to assess this.

\subsubsection{Identification of production constraints}

The process of developing the simulation model and establishing a viable base case scenario highlighted a number of bottlenecks in the initial production operations including the following:

\subsubsection{Sequential bogging and sampling for initial operational configurations}

Bogging and sampling was identified as a key production bottleneck for early operational configurations. Only one operation (either bogging or sampling) was permitted in each crows foot at a time for early configurations due to the potential for entrapment of other equipment in the event of a loader breakdown in a common access area. The inability to continue bogging operations while sampling in another crosscut often constrained the tonnes that could be bogged in a shift to below production targets in early simulation runs. As a result of this being identified as a bottleneck, sampling responsibility was placed on the loader operator to eliminate the interaction issue and improve loader efficiency.

\subsubsection{Bogging operations}

Bogging operations presented an occasional bottleneck if only one loader was operating in the panel compared to two operating. The average tonnes per operating hour during bogging increases with cave progression, as the location of the active rings get closer to the stockpiles and ore-passes. Figure 
6 shows the change in loader productivity in tonnes per operating hour with shift number (x-axis). The likelihood of bogging operations presenting as a production bottleneck with one loader working the panel therefore reduces with cave progression.

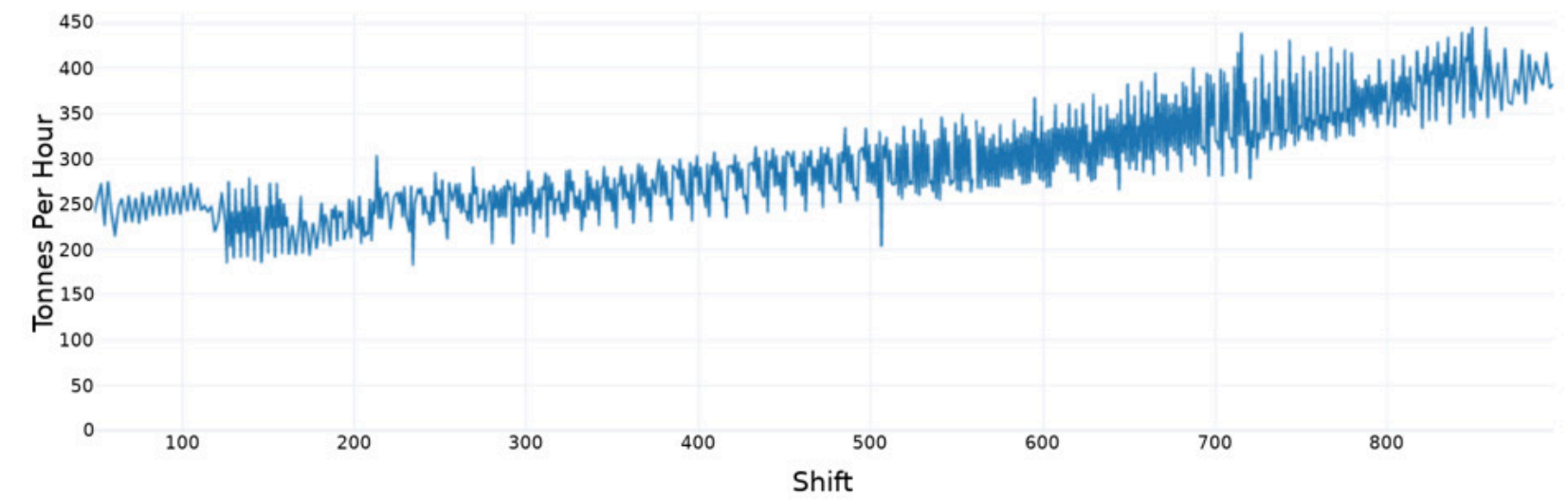

\section{Figure 6 Change in loader productivity with cave progression}

Initial iterations of the model stipulated that the tonnes are drawn evenly across available rings in 200 $\mathrm{t}$ increments (at which point sampling is required) to support an "even draw rate" strategy, rather than bogging all tonnes in a given ring in a single event. Results showed that this generated a constraint due to the frequency of bogger relocations to another crosscut causing inefficiencies, and that failure to complete bogging of rings can reduce the number of rings available to fire, thus reducing availability of broken stocks the following shift. As such, overall improvements to production operations could be achieved by prioritising completion of bogging tasks for critical rings by the end of the shift rather than adhere to an "even draw rate" strategy. Sampling frequency was increased to $500 \mathrm{t}$ as a result of identifying this constraint, and after an assessment of the statistical optimal sampling frequency.

While loader productivity varies depending on the distance between the active ring, the stockpile (or ore pass) and interactions with other activities, production tonnes per shift are primarily limited by the number of rings that were able to be fired in the previous shift. The production tonnes achieved in each shift for a simulation scenario with one loader operating is shown in Figure 7.

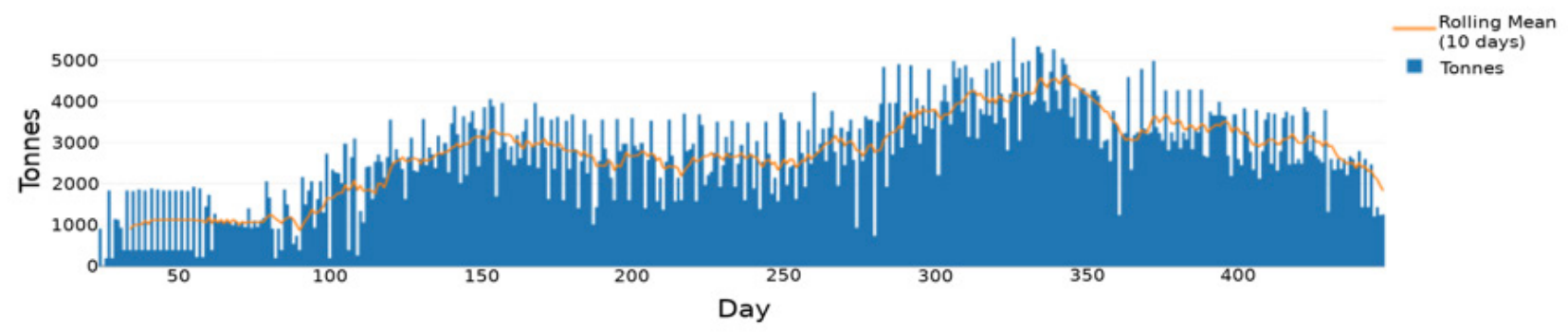

Figure 7 Production tonnes achieved in each shift

Restrictions on tonnes available for bogging in each shift as a result of an initial "panel-swapping" philosophy (described in section 2.1) and associated firing constraints are evident for early cave progression on the left side of the chart. For the case shown, more tonnes were bogged every second day during early cave progression, because one panel had access to more tonnes based on the geometry of the production level and chevron cave front. As a result of the simulation, the shift by shift panel swapping philosophy was removed prior to execution and is no longer a constraint. Instead, loaders and ancillary equipment may operate in separate crosscuts within the same crow's foot in any shift in circumstances where they are segregated from the tram route of the loader and not exposed to entrapment hazards.

\subsubsection{Ancillary operations}

Panel swap and cave front progression constraints were the primary bottleneck for production operations for the base case scenarios. The number of activities completed in each shift of an example simulation 
run is shown in Figure 8, with the shift number on the x-axis and the number of activities completed shown on the y-axis. Each colour in the vertical bar chart represents different activity. The large number of activities highlighted the criticality of managing interactions and effective shift planning to ensure that the bottleneck activities were not impeded.

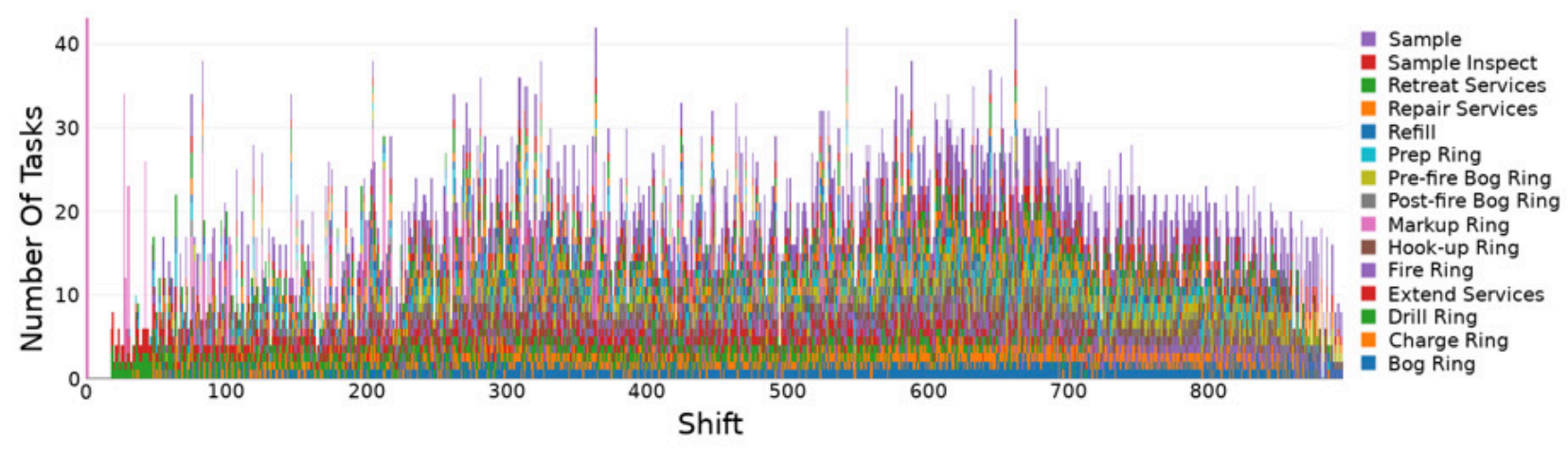

Figure 8 Number and type of tasks completed in each shift for a level

Analysis of simulation model results highlighted that short-term poor decisions or delays could have a significant impact on production not just for the current shift but often days later (e.g. no rings available to bog because a ring was not fired in time).

\subsubsection{Ring firing}

For the initial scenario configurations, hooking up of a ring was constrained to the last 30 min prior to the firing window. Analysis highlighted that this constraint often did not permit sufficient time to hook-up more than 3 rings per shift. Not all of the available rings were hooked up and production was constrained as a result. The simulation prompted the use of an electronic initiation system (hooked up to MS Detonators in the blast holes) to manage the risk associated with early hook-up, and as such hook-up of rings can now occur at any time during the shift, removing the hook-up constraints on production.

\section{Conclusions}

Discrete event simulation helped OZ Minerals in verifying the executability of the mine plan, and aided in the identification of bottlenecks, risks and opportunities within a production level. Key learnings included:

- The simulation identified that the forecast assumptions were appropriate and that production forecasts were achievable.

- Identification that certain operational philosophies were either flawed or not optimal, leading to both minor level design changes and changes to operating philosophy.

- Verification of resourcing requirements to achieve production forecasts, and the optimal number of loaders working on a level.

- Generally accepted concepts or conflicting opinions that could previously not be supported by data were able to be demonstrated / confirmed.

- Scenarios could be run to test different rate assumptions for activities to identify the constraining activities.

- In order to develop the simulation model, a discovery process was required which prompted more detailed discussions, thinking, design finalisations, and confirmation of preferred options (when faced with more than one). This was a good kickstart for the operational and technical teams to further understand how the level would function. 
This process also cemented that regardless of how optimised the level design is, and how well resourced the operation is, it is the decision making at the shift and hourly level that is ultimately going to drive the overall performance of the level, as the downstream effects of making a sub-optimal decision can lead to far greater consequences.

\section{Acknowledgement}

This paper summarises the extensive work of a small team from OZ Minerals and Polymathian, and the authors would like to acknowledge their efforts during the project and in preparing this paper.

\section{References}

Mooney, A, Grosser, H, Marsden, J \& Dunstan, G 2020, 'Carrapateena Crows Foot Level Layout - Safety in Design to Support Sublevel Cave Production Efficiency and Automation', Proceedings of MassMin 2020, Santiago, Chile. 